\title{
A qualitative analysis of patient-reported anticipated benefits of having elective surgery
}

\author{
David Yachnin, MSc • Emily Hladkowicz, MA, Ed (D) Daniel I. McIsaac, MD, \\ MPH, FRCPC
}

Received: 24 November 2020/Revised: 7 December 2020/Accepted: 7 December 2020/Published online: 6 January 2021

(C) Canadian Anesthesiologists' Society 2021

\section{To the Editor,}

Risk prediction before surgery is a commonly discussed and researched aspect of preoperative care. Accordingly, numerous risk prediction models have been developed and efforts have been undertaken to develop consensus around which outcomes should be routinely collected and studied in the perioperative period. Nevertheless, the patient decision-making and consent process requires that potential risks be discussed balanced with anticipated benefits of surgery. Interestingly, the benefits that patients

D. Yachnin, MSc

Department of Anesthesiology \& Pain Medicine, The Ottawa Hospital, Ottawa, ON, Canada

Ottawa Hospital Research Institute, Ottawa, ON, Canada

E. Hladkowicz, MA, Ed $(\bowtie)$.

Department of Anesthesiology \& Pain Medicine, The Ottawa

Hospital, Ottawa, ON, Canada

e-mail: emhladkowicz@toh.ca

School of Rehabilitation Therapy, Queens' University, Kingston, ON, Canada

Ottawa Hospital Research Institute, Ottawa, ON, Canada

D. I. McIsaac, MD, MPH, FRCPC

Department of Anesthesiology \& Pain Medicine, The Ottawa

Hospital, Ottawa, ON, Canada

Ottawa Hospital Research Institute, Ottawa, ON, Canada

School of Epidemiology \& Public Health, University of Ottawa, Ottawa, ON, Canada

Department of Anesthesiology \& Pain Medicine, University of Ottawa, Ottawa, ON, Canada hope to derive from elective surgery are poorly documented and tools to predict benefit are uncommon.

Recently, we developed and evaluated a patient-facing personalized risk communication tablet application to directly provide patients with their personalized perioperative risk profiles prior to their preoperative anesthesiology consultation. ${ }^{1}$ We asked each participant to self-report and rank up to three anticipated benefits of surgery (free-text). Therefore, the application's output provided to patients and their anesthesiologists to discuss during the consultation featured both personalized anticipated benefits and personalized quantitative risk estimates.

The protocol for our prospective before-and-after study that evaluated our risk communication application was registered (ClinicalTrials.gov: NCT03422133) and research ethics board approval was obtained (Ottawa Health Sciences Network Research Ethics Board Protocol \#20170737-01H; 27 February 2018). Collection of anticipated benefits that adult patients hoped to achieve from having elective non-cardiac surgery was prespecified, but the qualitative analysis of these data from the larger study, reported herein, was not. Self-reported anticipated benefits were analyzed thematically according to the methods of Braun and Clarke. ${ }^{2}$ First, we created a set of codes using an inductive approach. Next, two independent reviewers assigned codes to each reported benefit and reviewers sought consensus. The same process was repeated to organize the codes into larger themes. We used a semantic approach to coding because the benefits of surgery were input without greater context, making it difficult to infer any meaning beyond the literal benefits. A total of 214 benefits of surgery were analyzed from 93 participants. 
Table Anticipated benefits by surgery type

\begin{tabular}{|c|c|c|c|c|c|}
\hline Theme & $\begin{array}{l}\text { All surgeries }(n= \\
214)\end{array}$ & $\begin{array}{l}\text { General, thoracic, urology, } \\
\text { gynecology } \\
(n=70)\end{array}$ & $\begin{array}{l}\text { Orthopedic and spinal }(n= \\
88)\end{array}$ & $\begin{array}{l}\text { Vascular }(n= \\
27)\end{array}$ & $\begin{array}{l}\text { Other } \\
(n=29)\end{array}$ \\
\hline $\begin{array}{l}\text { Improving a health } \\
\text { condition }\end{array}$ & $69(32.2 \%)$ & $47(67.1 \%)$ & $3(3.4 \%)$ & $7(25.9 \%)$ & $12(41.4 \%)$ \\
\hline Curing cancer (subtheme) & $21(9.8 \%)$ & $17(24.3 \%)$ & $0(0 \%)$ & $0(0 \%)$ & $4(13.8 \%)$ \\
\hline Quality of life & $39(18.2 \%)$ & $11(16.9 \%)$ & $16(18.2 \%)$ & $5(18.5 \%)$ & $7(24.1 \%)$ \\
\hline Independence & $45(21 \%)$ & $2(2.9 \%)$ & $33(37.5 \%)$ & $8(29.6 \%)$ & $2(6.9 \%)$ \\
\hline Mobility (subtheme) & $38(17.8 \%)$ & $1(1.4 \%)$ & $29(33 \%)$ & $6(22.2 \%)$ & $2(6.9 \%)$ \\
\hline Physical fitness & $17(7.9 \%)$ & $2(2.9 \%)$ & $9(10.2 \%)$ & $3(11.1 \%)$ & $3(10.3 \%)$ \\
\hline $\begin{array}{l}\text { Gaining strength } \\
\text { (subtheme) }\end{array}$ & $3(1.4 \%)$ & $1(1.4 \%)$ & $2(2.3 \%)$ & $0(0 \%)$ & $0(0 \%)$ \\
\hline $\begin{array}{l}\text { Weight control } \\
\text { (subtheme) }\end{array}$ & $7(3.3 \%)$ & $1(1.4 \%)$ & $2(2.3 \%)$ & $1(3.7 \%)$ & $3(10.3 \%)$ \\
\hline $\begin{array}{l}\text { Physical activity } \\
\text { (subtheme) }\end{array}$ & $7(3.3 \%)$ & $0(0 \%)$ & $5(5.7 \%)$ & $2(7.4 \%)$ & $0(0 \%)$ \\
\hline Pain and sensation & $37(17.3 \%)$ & $5(7.1 \%)$ & $26(29.5 \%)$ & $4(14.8 \%)$ & $2(6.9 \%)$ \\
\hline Miscellaneous & $7(3.3 \%)$ & $3(4.3 \%)$ & $1(1.1 \%)$ & $0(0 \%)$ & $3(10.3 \%)$ \\
\hline
\end{tabular}

The benefits of surgery fell into six distinct themes: 1) improving a health condition, 2) quality of life, 3) independence, 4) physical fitness, 5) pain and sensation, and 6) miscellaneous. The Table shows the frequency of each theme and the relationship between themes and subthemes. The most frequent theme was improving a health condition (69/214 benefits). This theme was also most commonly first-ranked (46/93 benefits) and was supported by statements about improving general health (e.g., "live longer", "feel better") as well as specific conditions (e.g., "get rid of hernia", "removal of...tumours"). Curing cancer emerged as a subtheme within this group (21/69 benefits). Independence was the second most frequently identified anticipated benefit (45/ 214 benefits), often related to the mobility subtheme (38/ 45). Mobility benefits were typically associated with people having orthopedic procedures. Statements related to improving quality of life were also common (39/214), often focused on reducing stress, improving ability to do activities, increasing energy, and returning to a previous lifestyle. Pain and sensation (37/214) related benefits were identified across surgical disciplines, while physical fitness emerged as a less common but distinct theme (17/214) related to strength gain, weight control, and physical activity or recreation.

As patient-oriented research and patient-reported outcomes continue to gain prominence, understanding what patients hope to gain from perioperative care is of increasing importance. This qualitative thematic analysis of patient-reported anticipated benefits of surgery provides a structured synopsis of what Canadian patients at an academic hospital hope to achieve from having elective surgery. While patients' highest priority is to resolve existing health issues, they also greatly value impacts on their overall quality of life, independence, physical function, and pain control. These priorities should be strongly considered when discussing perioperative care and in designing future research.

Disclosures None.

Funding statement The study was supported through peerreviewed grants from The Ottawa Hospital Academic Medicine Organization and The Ottawa Hospital Department of Anesthesiology \& Pain Medicine.

Editorial responsibility This submission was handled by Dr. Hilary P. Grocott, Editor-in-Chief, Canadian Journal of Anesthesia.

\section{References}

1. Hladkowicz E, Yachnin D, Boland L, et al. Evaluation of a preoperative personalized risk communication tool: a prospective before-and-after study. Can J Anesth 2020; DOI: https://doi.org/ 10.1007/s12630-020-01809-y.

2. Braun V, Clarke V. Using thematic analysis in psychology. Qual Res Psychol 2006; 3: 77-101.

Publisher's Note Springer Nature remains neutral with regard to jurisdictional claims in published maps and institutional affiliations. 